\title{
Nonlinear analysis of RC cylindrical tank and subsoil accounting for a low concrete strength
}

\author{
Pawel M. Lewiński ${ }^{1, *}$ and Stawomir Dudziak ${ }^{1}$ \\ ${ }^{1}$ Building Research Institute (ITB), Filtrowa 1, 00-611 Warszawa, Poland
}

\begin{abstract}
The paper discusses deformational and incremental approaches to a nonlinear FE analysis of soil-structure interaction including the description of behaviour of the RC structure and the subsoil under shortterm loading. Two kinds of constitutive models for ground and structure were adopted for a nonlinear interaction analysis of the RC cylindrical tank with subsoil. The constitutive laws for concrete and subsoil were developed in compliance with the deformational and flow theories of plasticity. Moreover, a non-linear elastic-brittle-plastic analysis of RC axisymmetric structures using finite element iterative techniques is presented. The results of the two types of FE analysis of soil-structure interaction are compared taking into account a low concrete strength of tank structure.
\end{abstract}

\section{Introduction}

The subject of the paper is a new concept of a non-linear analysis of soil-structure interaction, including the description of the brittle and elastic-plastic behaviour of reinforced concrete structures, and the elastic-plastic behaviour of the subsoil. The analysis of the RC axisymmetric structure is performed with the use of the finite element iterative technique. The tensile cracking, the multiaxial compressive response of concrete and soil, and the yielding of steel reinforcement are the main non-linear effects studied. The present paper discusses two concepts of a nonlinear FE analysis of soil-structure interaction, including the description of the RC structure, one based on the deformation theory and the other - on the plastic flow theory of plasticity. This study analyses the impact of subsoil on stress resultants in the shell and ground slab of the RC cylindrical tank, as well as their redistribution which appears mainly due to cracks and, to some extent - uneven subsidence. Papers dealing with the finite element ultimate load analysis of thick-walled reinforced concrete structures subjected to radial pressure have been published since around 1968 in relation to its application for prestressed concrete reactor vessels. Likewise, widespread research were carried out on the subject of elastic foundations and soil-structure interaction. However, in the hitherto existing publications not all of the possible schemes of cracking patterns of RC axisymmetric structures have been exhausted and also with regard to RC tanks, it is rather difficult to refer to publications comprising numerical elastic-brittleplastic analysis and soil-structure interaction. Nevertheless, many papers deal with axisymmetric RC structures, with the paper by Phillips and Zienkiewicz [1] and the book

*Corresponding author: p.lewinski@itb.p1 
by Kotsovos and Pavlović [2] being among the first publications. In case of the total strain constitutive model, the analysis of soil-structure interaction was performed with the use of the FE direct iteration method, while in case of the plastic flow model, the Newton-Raphson method was applied. The results of the analysis indicate that in the case of structural concrete and subsoil of normal strength, the scope of redistribution is not very large, whereas in the case of weak concrete or subsoil the redistribution range is quite wide. This analysis shows an increase in radial displacements of the shell, as well as a rise in hoop steel stresses in relation to particularly weak structural concrete.

\section{Material modelling}

\subsection{Failure criteria for concrete and cracking patterns}

In case of the total strain constitutive model for concrete the ultimate surface proposed by $\mathrm{J}$. Podgórski [3] was implemented. In the failure criterion assumed herein, the basic parameters can be written in the following manner:

$r(\varphi)=\sqrt{2 J_{2}}=[P(J)]^{-1}, P(J)=\cos [(\arccos (\alpha J)) / 3-\beta], J(\varphi)=\cos 3 \varphi=\frac{3 \sqrt{3} J_{3}}{2 J_{2}^{3 / 2}}$,

where $\alpha$ and $\beta$ are constants fulfilling the following inequalities: $0 \leq \alpha \leq 1$ and $0 \leq \beta \leq \pi / 6$. The parameters describing the proportion of radius $r$ for different $\varphi$ angles are as follows:

$$
\lambda=r\left(\varphi=0^{\circ}\right) / r\left(\varphi=60^{\circ}\right) \quad \vartheta=r\left(\varphi=30^{\circ}\right) / r\left(\varphi=60^{\circ}\right) .
$$

According to Podgórski [3], $\alpha$ and $\beta$ coefficients from equation (1) can be derived by means of iteration. However, the equations for the direct calculation of these parameters were proposed by Lewiński $[4,5]$. From the set of equations (1) and (2) for $\varphi=0^{\circ}, 30^{\circ}, 60^{\circ}$ the trigonometric equations can be obtained which can be solved with regards to $\alpha$ and $\beta$ :

$$
\alpha=\sin 3 \alpha_{0}, \beta=\frac{\pi}{6}-\arctan \left(\frac{\vartheta(1-\lambda)}{2 \lambda \sin \alpha_{0}}\right), \text { where } \alpha_{0}=\arccos \left(\frac{\vartheta}{2}\left(1+\frac{1}{\lambda}\right)\right)
$$

J. Podgórski confirmed the usefulness of the above expressions [6]. Assuming that in the octahedral set of coordinates the meridian of ultimate surface is described by a parabolic equation, the following equation for the failure surface for concrete was used (see Fig. 1a):

$$
F\left(\sigma_{o c t}, \tau_{o c t}, \varphi\right)=\sigma_{o}-C_{0}+C_{1} P(J) \tau_{o}+C_{2} \tau_{o}^{2}=0
$$

The failure surface (JP) described by the equations (1) and (4) is presented in Fig. 1a) (the inner blue surface). The values of $C_{0}, C_{1}, C_{2}, \alpha$ and $\beta$ coefficients can be derived on the basis of experimental data, utilizing the following strengths of concrete: uniaxial tensile strength $f_{t}$, uniaxial compressive strength $f_{c}$, biaxial compressive strength $f_{c c}\left(\sigma_{1} / \sigma_{2}=1\right)$, biaxial compressive strength $f_{o c}\left(\sigma_{1} / \sigma_{2}=2\right)$, triaxial tensile strength $f_{t}$. According to the tests performed by Kupfler et al. [7]: $f_{c c}=1.1 f_{c}$ and $f_{o c}=1.25 f_{c}$ were assumed. The values of $C_{0}$, $C_{1}$ and $C_{2}$ coefficients from the equation (4) can now be written in the following way:

$$
C_{0}=f_{t}, C_{1}=\frac{\sqrt{2}}{P(\varphi=0)}\left[1-\frac{3}{2} \frac{\left(f_{t} / f_{c c}\right)}{\left(f_{c c} / f_{t}-1\right)}\right], C_{2}=\frac{9}{2} \frac{f_{t} / f_{c c}}{f_{c c}-f_{t}}
$$


Such defined 5-parameter failure surface for concrete, despite its apparent complexity, poses no problem with numerical implementation and complies well with experimental results. In the tensile zones of concrete, cracking is modelled by introducing five types of specific cracking patterns, including radial cracks and circumferential cracks, sheared both parallel and perpendicular to the cracks. In case of the incremental FE analysis, the "Smeared Cracking" (SC) material model [8] was adopted for concrete. The two aforementioned failure surfaces for concrete which are interpenetrating, are presented in Fig. 1a): $J P$ (blue colour) and $S C$ (green colour). In case of $S C$ material model two intersecting conical surfaces are applied (see the axial cross-section of $S C$ failure surface in Fig. 4a) - a thin blue line).
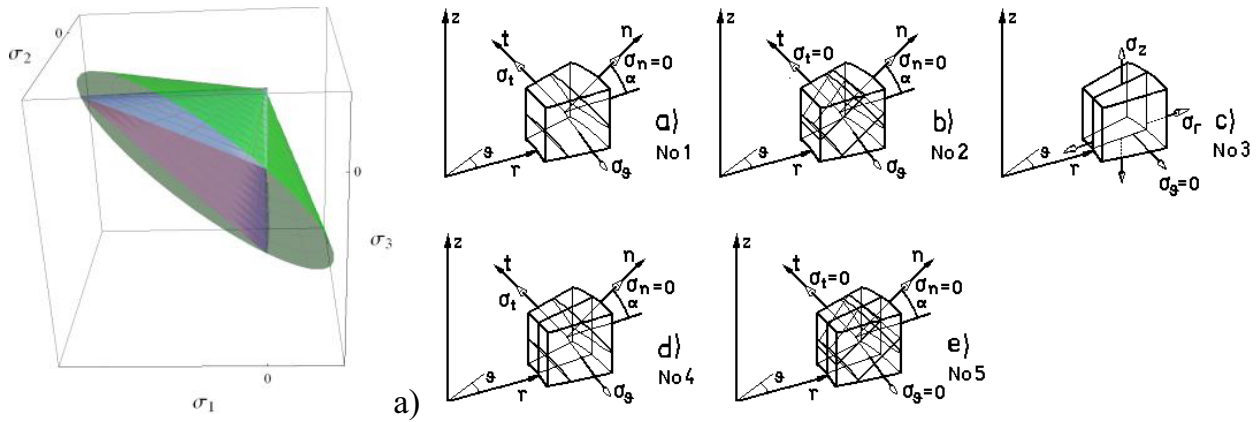

a)
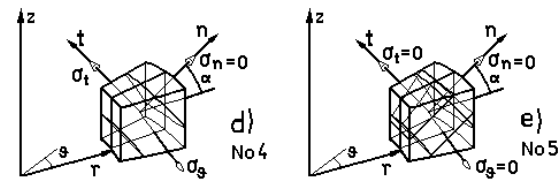

b)

Fig. 1. Cracking criteria: a) failure surfaces: $J P$ - blue colour, $S C$ - green colour, b) cracking patterns of RC axi-symmetric structure.

\subsection{Behaviour of concrete and soil}

The total strain analysis is based on the deformation theory of plasticity. The plastic deformation model is based on deviatoric and hydrostatic components of stress and strain. This implies that the concrete remains isotropic under multiaxial stress. Thus the octahedral-based elastoplastic model is utilized by assuming the secant bulk modulus $K_{S}$ and the secant shear modulus $G_{S}$ to be functions of the volumetric and the octahedral strains. A model similar to this (but additionally sensitive to lateral pressure) is adopted for the description of the subsoil. In the present paper the original description of Kupfer and Gerstle [7] has been modified by using corrections with respect to $\gamma_{\text {oct }}$ :

$$
\begin{aligned}
& \frac{G_{S}}{G_{0}}=f_{c} \frac{\sqrt{f_{c}^{2}+4 a b G_{0}^{2} \gamma_{o c t}^{2}}-f_{c}}{2 a b G_{0}^{2} \gamma_{o c t}^{2}}-\gamma_{o c t} \cdot \omega \cdot\left(\alpha_{1} \cdot \gamma_{o c t}+\alpha_{2} \cdot \omega_{23}\right) \\
& \frac{K_{S}}{K_{0}}=\frac{G_{S}}{G_{0}}-\gamma_{o c t} \cdot \omega \cdot\left(\alpha_{1} \cdot \gamma_{o c t}+\alpha_{2} \cdot \omega_{23}\right),
\end{aligned}
$$

where $a, \alpha_{1}, \alpha_{2}, \omega$ and $\omega_{23}$ are the numeral coefficients dependent on $\alpha_{m}$, where $\alpha_{m}=$ $1000 \cdot f_{c}^{\prime} / E_{c}$ for concrete and $100 \cdot R_{c} / E_{0}$ for soil. For concrete $b=1$, while for soil $b$ means an empirical relationship (taking into account the value of lateral pressure). In the performed numerical analysis, the value of lateral stress $\sigma_{r}$ was taken into account instead of lateral pressure. $K_{S}$ and $G_{S}$ modules do not depend on the third strain tensor invariant due to the limitations imposed by the theory of the representations of tensor functions and Noll conditions (see [9]). For the incremental FE analysis, the "Smeared Cracking" elastic plastic model [8] was adopted for concrete. Elastic - perfectly plastic material with the Coulomb-Mohr yield condition and the non-associated flow rule was applied for the soil. 


\subsection{Models for reinforcing steel and tension stiffening}

Both the tension stiffening effect and the shear retention are taken into account, wherever the former is included, assuming that additional stress is carried by the reinforcement steel. In case of the deformational model of reinforcing steel the tension stiffening effect was adopted on the assumption that additional stress is carried by rebars. The stress-strain relationship for reinforcing steel is assumed as elastic-rigid plastic. The concept of modelling the tension stiffening by the control of reinforcing bars' stiffness is not quite new (see the paper [10]). The influence of the reinforcement bond in cracked concrete on shell or slab deformation was taken into account by the tension stiffening approach according to the provisions of Eurocode 2 [11]. The Expression (7.18) given in this standard can also relate to the strains in reinforcing steel in the arbitrary $i$ direction and take the form:

$$
\varepsilon_{s m i}=\zeta \varepsilon_{s i}+(1-\zeta) \varepsilon_{u c r i}
$$

where:

$\varepsilon_{s m i}, \varepsilon_{u c r i}, \varepsilon_{s i}=$ the mean strain in the reinforcement (according to EC2 [11]) between the cracks in $i$ direction (for intermediate bond of rebar) and the strains in the reinforcement calculated for the uncracked and fully cracked conditions, respectively, $\zeta=$ a distribution coefficient allowing for tension stiffening (see Eqn. (7.19) in EC2 [11]).

Therefore, the tension stiffening coefficient can be introduced to control the effective elasticity modulus of the reinforcing bars $\left(\bar{E}_{s i}=E_{s} / \psi_{s i}\right)$ as follows:

$$
\psi_{s i}=\varepsilon_{s m i} / \varepsilon_{s i}=\zeta+(1-\zeta)\left(\varepsilon_{u c r i} / \varepsilon_{s i}\right)
$$

Substituting the coefficient $\zeta$ by the Expression (7.19) (see EC2 [11]), after some transformations, the following formula is obtained:

$$
\psi_{s i}=1-\beta\left(1-\varepsilon_{u c r i} / \varepsilon_{s i}\right)\left(\sigma_{s r i} / \sigma_{s i}\right)^{2}
$$

where in turn the steel stress in cracked section $\sigma_{s i}$ can be replaced by the ratio of its mean value and the tension stiffening coefficient: $\sigma_{s m i} / \psi_{s i}$, as the stress $\sigma_{s m i}$ is directly available from the iterative numerical analysis. The ratio of the strains $\varepsilon_{u c r i} / \varepsilon_{s i}$ can also be determined in a numerical way and coefficient $\beta$ can be assumed according to EC2 [11]. After some rearrangements the coefficient $\psi_{s i}$ can be formulated depending on mean stress $\sigma_{s m i}[5]$.

For the incremental Abaqus model [9] the reinforcement was included with the "Rebar" option. An elastic-plastic analysis with hardening steel model was used.

\section{Computational examples}

In the paper, axisymmetric elements are used throughout. In the case of the first model, the analysis of soil-structure interaction was conducted by an own programme (namely an expanded FEAP version). That analysis was performed using the Lagrange quadrilateral isoparametric element. The second model was built using Abaqus software with 3-node axisymmetric shell elements (SAX2, for structure) and 8-node axisymmetric continuum elements (CAX8R, for soil). The analysis of the impact of low-strength concrete in the tank on the soil-structure interaction was performed taking into account the characteristics of the concrete given below. The purpose of this analysis is to show the impact of this significant, although an adverse factor on the redistribution of stress resultants. The computational example of a nonlinear FE analysis of the RC tank with cylindrical shell (with the inner radius of $6.90 \mathrm{~m}$, height of $5 \mathrm{~m}$, thickness of $20 \mathrm{~cm}$ ) connected to the ground slab (with the 
depth of $20 \mathrm{~cm}$ ) supported on elasto-plastic subsoil is given. For the deformational analysis the following properties of concrete were adopted: $f_{c}=20 \mathrm{MPa}, f_{c t}=0.4 \mathrm{MPa}, E_{c}=20 \mathrm{GPa}$, $v_{c}=0.167$; subsoil strength: $R_{c}=0.2 \mathrm{MPa}, E_{0}=30 \mathrm{MPa}, v_{0}=0.35$; steel reinforcement was adopted as $f_{y}=355 \mathrm{MPa}, E_{s}=210 \mathrm{GPa}$. The vertical reinforcement of the shell: $\varnothing$ $16 / 20 \mathrm{~cm}$ and horizontal reinforcement: $\varnothing 20 / 20 \mathrm{~cm}$ were adopted on both sides, while in the ground slab - the radial and peripheral reinforcement: $\varnothing 18 / 13 \mathrm{~cm}$. Near the junction of the shell with the slab, the hoop bars are concentrated: $\varnothing 20 / 12 \mathrm{~cm}$. The following soil parameters were introduced for the incremental FE analysis: $E=30 \mathrm{MPa}, v=0.35, c^{\prime}=25$ $\mathrm{kPa}, \varphi^{\prime}=20^{\circ}, \psi=0^{\circ}$. The assumed soil parameters correspond to clay. For the incremental analysis the concrete properties were adopted as specified above. In regards to reinforcement, it was assumed that $f_{u}=510 \mathrm{MPa}$.

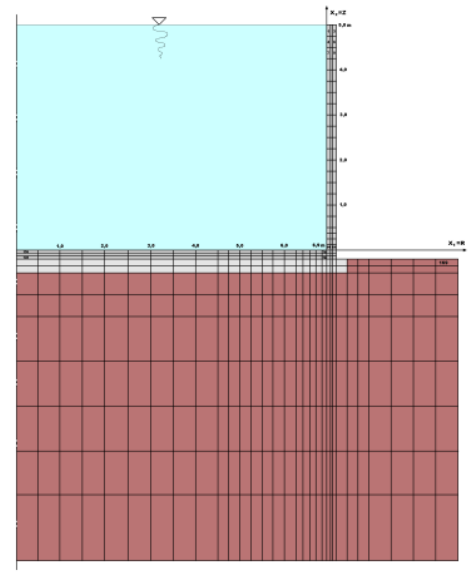

a)

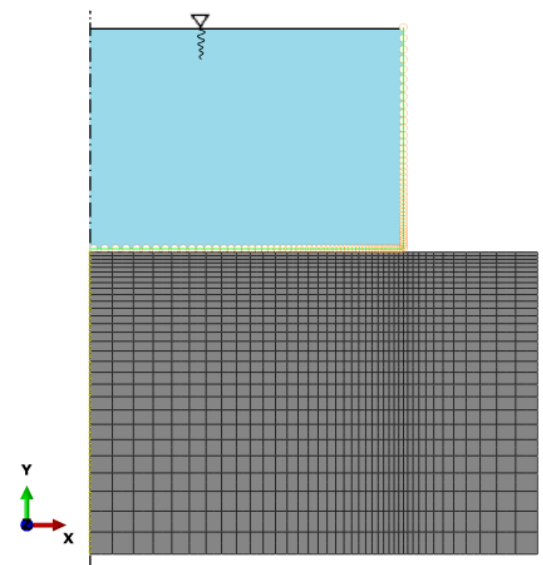

b)

Fig.2. Computational schemes of the tank structure; vertical cross-sections showing division into the finite elements and the liquid level for both models: a) deformational, b) incremental.

\section{Results and conclusions}

The comparative analysis was performed for both incremental and small-strain stiffness constitutive models. Analytical results are presented in Figures 3 to 9.

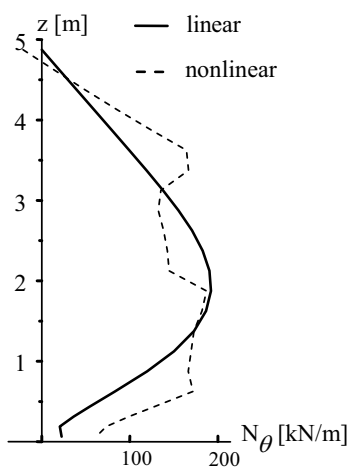

a)

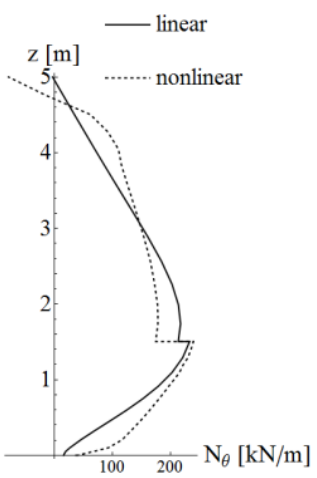

b)

Fig. 3. Circumferential forces in tank wall due to hydrostatic pressure for both models:

a) deformational, b) incremental. 
The diagrams of internal forces, $N_{\vartheta}$ and $M_{z}$, in the shell in the elastic state and physically non-linear stage are shown in Fig. 3 and Fig. 5, respectively. The range of redistribution of internal forces obtained here is quite significant compared to the case of normal strength concrete. The redistribution of the forces $N_{\vartheta}$ and $M_{z}$ is a result of cracks in the shell when the concrete strength is lower than normal, as the shell is more susceptible to deformation. Moment at the connection of the shell and the slab is smaller than in case of normal strength concrete due to the cracking near the joint. The chart of circumferential forces $N_{\vartheta}$ shows the additional loading of non-cracked zones and unloading of cracked zones (due to decrease in stiffness), especially for the more restrictive $J P$ criterion (Fig. 4a).
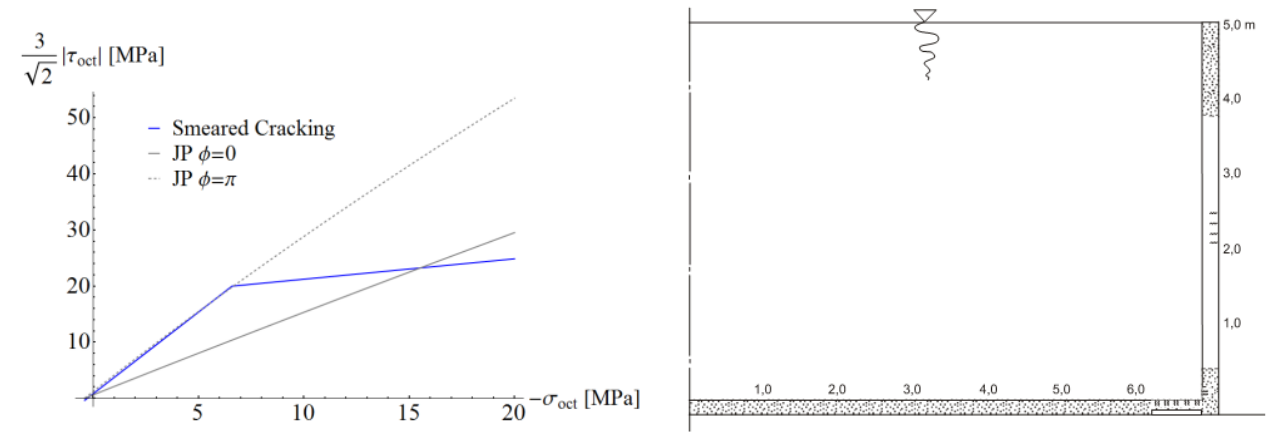

a)

b)

Fig. 4. Cracking phenomenon: a) failure criteria, b) cracks in tank structure for deformational model.
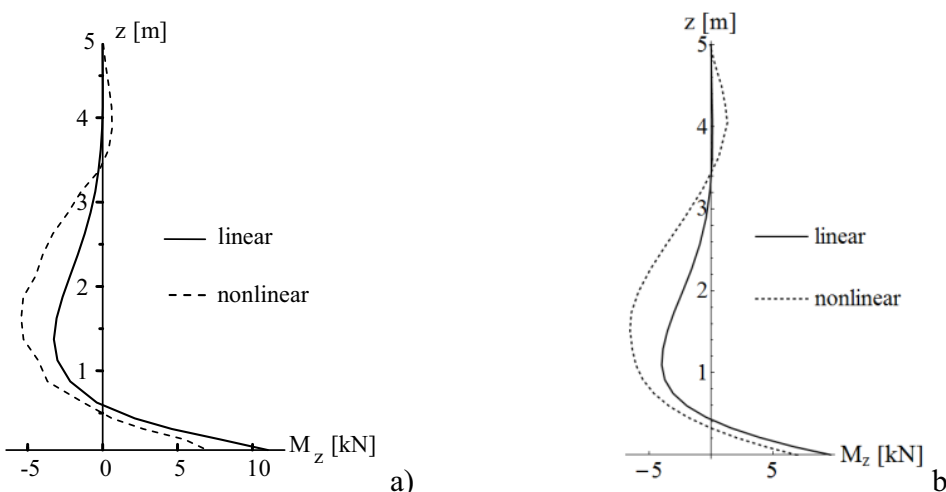

Fig. 5. Bending moment in the tank wall due to hydrostatic pressure for both models: a) deformational, b) incremental.
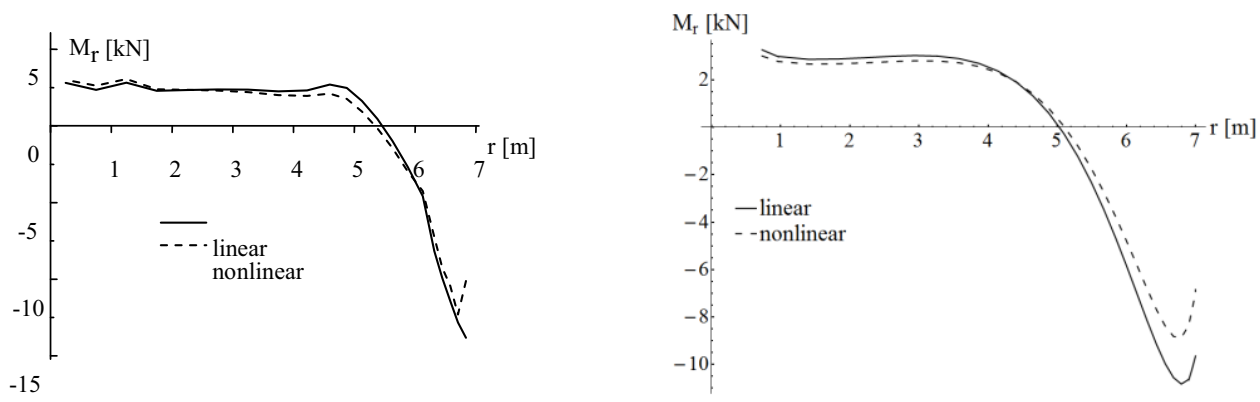

a)

b)

Fig. 6. Radial moments in the slab for the a) deformational and b) incremental model. 

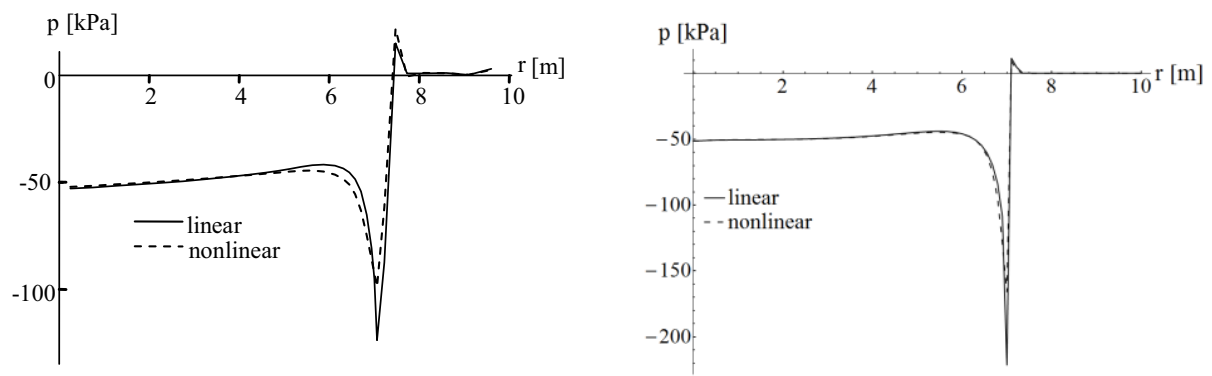

a)

b)

Fig. 7. Distributions of the ground reaction for both models: a) deformational, b) incremental.

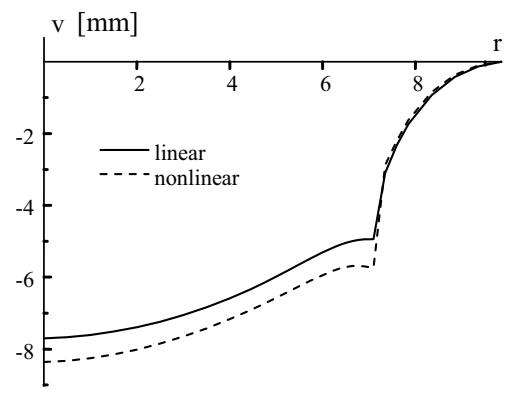

$[\mathrm{m}]$

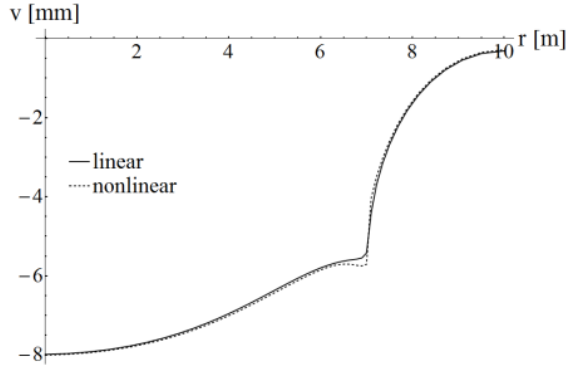

a)

b)

Fig. 8. Distributions of the ground subsidence for the: a) deformational and b) incremental model.

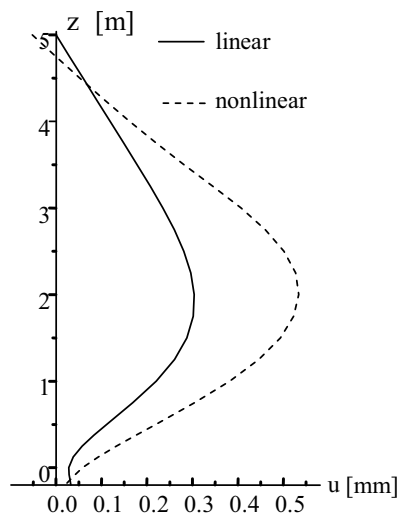

a)

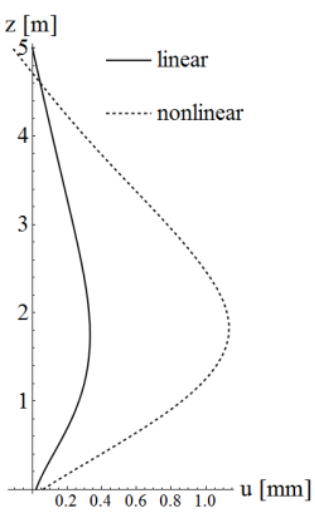

b)

Fig. 9. Distributions of the radial displacements of the tank shell for the: a) deformational and b) incremental model.

The diagrams of bending moments, $M_{r}$, in the ground slab in the elastic state and physically non-linear stage are shown in Fig. 6. In this case, the slab moment $M_{r}$ at the connection of the slab and the shell is smaller than in the elastic stage. Different values of bending moments at the connection of the slab with the cylindrical shell also result from nonlinear behaviour of the ground under the slabs in both cases (see Fig. 8) as well as stress redistribution in the substrate soil. The subsoil reaction is also redistributed due to the development of plastic strains in the soil (see Fig. 7). Different values of subsoil reactions in elastic solutions for both models are mainly a result of different FE discretisiations. A significant increase in radial displacements of the tank shell is observed due to the cracks 
(from a maximum of $0.304 \mathrm{~mm}$ in the elastic stage to $0.534 \mathrm{~mm}$ in the inelastic stage for the deformational model and to more than $1 \mathrm{~mm}$ for the incremental model, see Fig. 9) and the increase of stress in the circumferential reinforcement of the shell. Different results obtained for both models (see Fig. 3 and Fig. 9) are linked with the differences of the shapes of both limiting surfaces (see Fig. 1 a) and Fig. 4a), which affects the different cracking patterns. During the deformational analysis there were registered cracking patterns № 3 and № 1 in the walls of the tank shell and, occasionally, cracking patterns № 1 and № 2 in the ground slab. Due to the weak concrete the scope of cracking is very extensive. Vertical cracks in the cylindrical tank shell designated by the iterative FE analysis, pass through (cracking pattern № 3) and occur in the area of the shell from $0.375 \mathrm{~m}$ to $3.75 \mathrm{~m}$ above the ground slab. The horizontal and skew cracks in the vertical cross-section occurred in the walls of the tank only in their lower part (near the connection with the ground slab), while the cracks in the ground slab resulting from the bending occurred in the area of the connection with the shell and they pass only through a part of the cross-section. As a result of the deformational analysis there were registered mainly the cracks extending from the top of the ground slab according to cracking pattern № 1 (occasionally № 2) over a distance of $0.9 \mathrm{~m}$ from the connection with the tank wall, and, furthermore, the cracks extending from the bottom of the slab according to the cracking pattern № 3, resulting from the bending in circumferential direction, occurring at the distance $0.4 \mathrm{~m}$ from the junction in the part of the ground slab along the edge.

The results of the analysis indicate that in the case of structural concrete and subsoil of normal strength, the scope of redistribution is not very large, whereas in the case of weak concrete (or subsoil) the redistribution range is quite wide, which is accompanied by the increase in the horizontal displacements of the shell and the width of the vertical cracks.

\section{References}

1. D. V. Phillips, O. C. Zienkiewicz, Proc. Inst. Civ. Engrs, Part 2, 61 (3), 59 (1976)

2. M. D. Kotsovos, M. N. Pavlović, Structural concrete. Finite-element analysis for limitstate design (Thomas Telford Services Ltd, London, 1995)

3. J. Podgórski, Arch. Mech., 36 (3), 323 (1984)

4. P. M. Lewiński, $2^{\text {nd }}$ Int. Sci. AMCM Conference, Lódź, Poland, 405 (1996)

5. P. M. Lewiński, Analysis of interaction of reinforced concrete cylindrical tanks with subsoil (in Polish) (ITB, Warsaw, 2007)

6. J. Podgórski, Mechanics and Control, 30 (4), 229 (2011)

7. H. B. Kupfer, K. H. Gerstle, J. Engng Mech. Div., Proc. ASCE, 99 (EM4), 853 (1973)

8. Abaqus Theory Manual, Ver. 6.12 (Dassault Systèmes, 2012)

9. S. Jemioło, A. Szwed, Logistyka, 6, CD (2009)

10. H. Floegl, H. A. Mang, Journal of the Structural Division, Proc. ASCE, 108 (ST12), 2681 (1982)

11. EN 1992-1-1:2004 Eurocode 2: Design of concrete structures - Part 1-1: General rules and rules for buildings (2004) 